\title{
EVALUACIÓN DEL DESEMPEÑO DOCENTE Y LAS HABILIDADES PARA LA VIDA DE LOS ESTUDIANTES DEL NIVEL SECUNDARIO DE LA INSTITUCIÓN EDUCATIVA PRIVADA PAMER, LOS OLIVOS - LIMA NORTE, 2017
}

\author{
Danny Alexander LEÓN ORTIZ ${ }^{1}$ \\ Ofelia Carmen SANTOS JIMÉNEZ ${ }^{2}$ \\ https://orcid.org/0000-0003-1294-0641
}

\begin{tabular}{|lcc|}
\hline Recibido & $:$ & 20.03 .2020 \\
Aceptado & $:$ & 02.06 .2020 \\
Publicado & $:$ & 06.07 .2020 \\
\hline
\end{tabular}

RESUMEN: La presente investigación trata en determinar la influencia que existe entre la evaluación del desempeño docente y las habilidades para la vida en los estudiantes del nivel secundario de la Institución Educativa Privada PAMER Los Olivos - Lima Norte, 2017. En base a la revisión de la literatura existente se establece tres dimensiones para la variable independiente Estrategias didácticas, Capacidades pedagógicas, Responsabilidad de funciones laborales. Para la variable dependiente se establece 3 dimensiones las Habilidades Sociales, Cognitivas y para Manejar Emociones. Es una investigación de Enfoque: Cuantitativo de tipo básica, de Nivel Descriptiva-causal. Diseño: no experimental de corte transversal. Para la recolección de datos se utilizó un cuestionario a directivos, y estudiantes. Para la contratación de hipótesis se usó la correlación de R-Spearman entre las variables, los resultados indican que la evaluación del desempeño docente influye significativamente en las habilidades para la vida en los estudiantes del nivel secundario de la Institución Educativa Privada PAMER Los Olivos - Lima Norte, 2017 y la correlación es significativa y alcanza un nivel de 0.783 que corresponde a un nivel de correlación positiva moderada.

Palabras claves: Evaluación, desempeño docente, habilidades, estudiantes

\section{Evaluation of the teaching performance and life skills of secondary school students at the Private Educational Institution PAMER, Los Olivos - Lima Norte, 2017}

\begin{abstract}
The present investigation tries to determine the influence that exists between the evaluation of the educational performance and the abilities for the life in the students of the secondary level of the Private Educational Institution PAMER Los Olivos - Lima North, 2017. Based on the review of the literature There are three dimensions for the independent variable Teaching strategies, Pedagogical abilities, Responsibility of labor functions. For the dependent variable, 3 dimensions are established, social, cognitive and emotional skills. It is an investigation of Focus: Quantitative of basic type, of Descriptive-causal Level. Design: non-experimental cross-section. For the data collection, a questionnaire was used to managers and students.For the contracting of hypotheses the correlation of R-Spearman between the variables was used, the results indicate that evaluation of teacher performance significantly influences life skills in students of the secondary level of the Private Educational Institution PAMER Los Olivos - Lima Norte, 2017 and the correlation is significant and reaches a level of 0.783 that corresponds to a moderate positive correlation level.
\end{abstract}

Keywords: Assessment, teacher performance, skills, students.

\footnotetext{
${ }^{1}$ Universidad Nacional Mayor de San Marcos. Email: danny.leonortiz@gmail.com

${ }^{2}$ Universidad Nacional Mayor de San Marcos. Email: ofelia.santos@unmsm.edu.pe
} 


\section{INTRODUCCIÓN}

Toda sociedad moderna alcanza a concebirse como tal con ciudadanos con valores bien cimentados, comprometidos con su entorno y con alto sentido ético. El artículo $29^{\circ}$ de la Ley General de Educación establece que "La educación básica está destinada a favorecer el desarrollo integral del estudiante, el despliegue de sus potencialidades y el desarrollo de capacidades, conocimientos, actitudes y valores fundamentales que la persona debe poseer para actuar adecuada y eficazmente en los diversos ámbitos de la sociedad".

Dentro de esta perspectiva, nuestra investigación pretende demostrar la relación que existe entre la evaluación del desempeño docente y las habilidades para la vida en los estudiantes del nivel secundario de la Institución Educativa Privada PAMER Los Olivos - Lima Norte.

Los informes e incidencias actitudinales reportados por los entes educativos de este plantel han identificado diversos problemas con los estudiantes del nivel secundario: problemas de conducta, falta de motivación, escasa comunicación afectiva, entre otros. El diseño curricular de esta institución se basa en el enfoque de Habilidades para la Vida, las cuales sirven para que los alumnos logren enfrentar exitosamente situaciones complicadas en la vida cotidiana. Dichas habilidades se fomentan por medio de entrenamiento intencional o experiencia directa a través del modelado o la imitación.

Las habilidades consideradas en esta investigación demostrarán que son básicas para que el estudiante enfrente de manera adecuada los desafíos en los distintos planos de la vida: familia, escuela, amigos y otros espacios de interacción.

En tal sentido, y en procura de fomentar dichas habilidades, es fundamental el rol del docente y poder observar su actuar en las aulas. Para ello, se requiere una evaluación respecto al desempeño docente y así garantizar que poseen la capacidad pedagógica, conciencia ética y moral suficientemente desarrolladas. Tal evaluación, incluidas la autoevaluación y coevaluación, se logra por medio de análisis y reflexión pedagógicos que deben ser compatibles con las prácticas que la comunidad educativa considera adecuadas.

En efecto, ambos factores son esenciales; es importante desarrollar en los estudiantes habilidades que les permitirán desenvolverse con éxito en la vida adulta. Sin embargo, se evidencia el bajo nivel de dichas habilidades en los estudiantes y se advierte el peligro que puede conllevar ello para su futuro. Entonces, probablemente, los docentes, quienes están encargados de fortalecer estas, no realizan cabalmente esta función y, por ende, no están siendo debidamente evaluados en sus desempeños.

Por ello, una adecuada evaluación del desempeño de los docentes en esta institución educativa servirá para verificar que estén realizando actividades orientadas al fortalecimiento de dichas habilidades. La investigación puede ser de utilidad para la comunidad educativa, en donde docentes y estudiantes sean conscientes de los roles que cumplen para alcanzar el desarrollo pleno de sus roles en la sociedad

\section{DESARROLLO}

\section{Objetivo general}

Determinar la influencia que existe entre la evaluación del desempeño docente y las habilidades para la vida de los estudiantes del nivel secundario de la Institución Educativa Privada PAMER, Los Olivos Lima Norte, 2017.

\section{Objetivos Específicos}

- Demostrar que las estrategias didácticas influyen en las habilidades para la vida de los estudiantes del nivel secundario de la Institución Educativa Privada PAMER, Los Olivos - Lima Norte, 2017. 
- Demostrar que existe influencia de las capacidades pedagógicas en las habilidades para la vida de los estudiantes del nivel secundario de la Institución Educativa Privada PAMER, Los Olivos - Lima Norte, 2017.

- Demostrar que la responsabilidad de funciones laborales influye en las habilidades para la vida de los estudiantes del nivel secundario de la Institución Educativa Privada PAMER, Los Olivos - Lima Norte, 2017.

\section{Metodología}

\section{Tipo de investigación}

- $\quad$ El desarrollo de la investigación es para conocer cómo la evaluación del desempeño docente influye en las habilidades para la vida de los estudiantes del nivel secundario. Se tipifica según los siguientes criterios.

- $\quad$ Por el nivel de profundidad de estudio, será descriptiva causal porque se detallará la manera de como la evaluación del desempeño docente influye en las habilidades para la vida en los estudiantes.

- Por el método de estudio de las variables, es una investigación cuantitativa ya que se obtendrán datos numéricos categorizados en las variables.

- $\quad$ Es una investigación de campo con un diseño NO experimental.

Para probar la hipótesis de la presente investigación se ha elegido la estrategia bivariada explicativa porque se ha empleado dos variables con el objetivo de indagar acerca del efecto de la variable independiente sobre la dependiente establecer la relación de causalidad entre la variable independiente y la dependiente.

Esquematizando, matemáticamente tenemos:

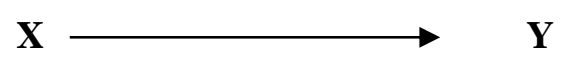

\section{X: EVALUACIÓN DEL DESEMPEÑO DOCENTE Y: HABILIDADES PARA LA VIDA}

\section{Discusión de Resultados}

Los resultados se muestran descriptivamente mediante tablas que comparan las frecuencias porcentuales de la variable evaluación del desempeño docente y las habilidades para la vida de los estudiantes.

\section{Variable: Evaluación del Desempeño Docente \\ Dimensión: Estrategia Didáctica - Resultados Encuesta Directivos}

\section{Cuadro $\mathbf{N}^{\circ} 1$}

\begin{tabular}{|c|l|l|l|l|l|}
\hline \multicolumn{2}{|c|}{} & Frecuencia & Porcentaje & $\begin{array}{l}\text { Porcentaje } \\
\text { válido }\end{array}$ & $\begin{array}{l}\text { Porcentaje } \\
\text { acumulado }\end{array}$ \\
\hline \multirow{3}{*}{ Válido } & REGULAR & 1 & 50,0 & 50,0 & 50,0 \\
\cline { 2 - 6 } & BUENA & 1 & 50,0 & 50,0 & 100,0 \\
\cline { 2 - 6 } & Total & 2 & 100,0 & 100,0 & \\
\hline
\end{tabular}

Fuente. Datos procesados mediante el software SPSS 24 y Excel

Elaborado: Por el responsable de la investigación. 


\section{Figura $\mathbf{N}^{\circ} 1$}

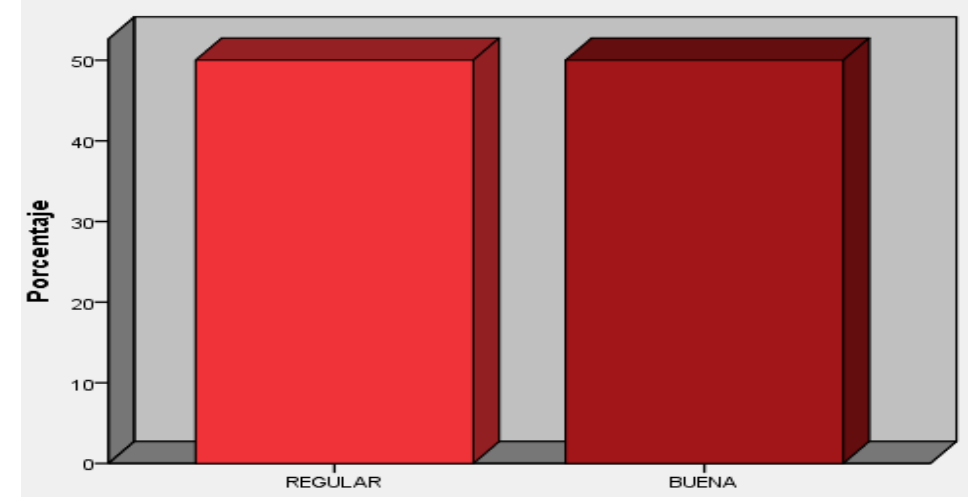

DIMENSIÓN: Capacidades Pedagógicas - Resultados Encuesta Directivos Cuadro $\mathbf{N}^{\circ} 2$

\begin{tabular}{|c|l|c|c|c|c|}
\hline \multicolumn{2}{|c|}{} & Frecuencia & Porcentaje & $\begin{array}{c}\text { Porcentaje } \\
\text { válido }\end{array}$ & $\begin{array}{c}\text { Porcentaje } \\
\text { acumulado }\end{array}$ \\
\hline \multirow{3}{*}{ Válido } & REGULARES & 1 & 50,0 & 50,0 & 50,0 \\
\cline { 2 - 6 } & BUENAS & 1 & 50,0 & 50,0 & 100,0 \\
\cline { 2 - 6 } & Total & 2 & 100,0 & 100,0 & \\
\hline
\end{tabular}

Fuente. Datos procesados mediante el software SPSS 24 y Excel Elaborado: Por el responsable de la investigación.

\section{Figura $\mathbf{N}^{\circ} 2$}

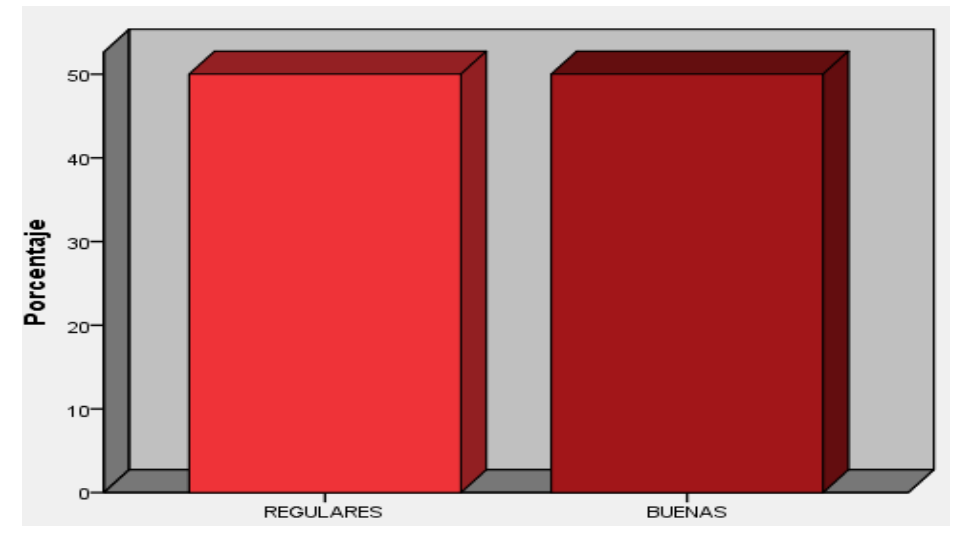

DIMENSIÓN: Responsabilidad de Funciones Laborales - Resultados Encuesta Directivos Cuadro $\mathbf{N}^{\circ} 3$

\begin{tabular}{|c|l|c|c|c|c|}
\hline \multicolumn{2}{|c|}{} & Frecuencia & Porcentaje & $\begin{array}{c}\text { Porcentaje } \\
\text { válido }\end{array}$ & $\begin{array}{c}\text { Porcentaje } \\
\text { acumulado }\end{array}$ \\
\hline \multirow{4}{*}{ Válido } & $\begin{array}{l}\text { POCO } \\
\text { RESPONSABLE }\end{array}$ & 1 & 50,0 & 50,0 & 50,0 \\
\cline { 2 - 6 } & RESPONSABLE & 1 & 50,0 & 50,0 & 100,0 \\
\cline { 2 - 6 } & Total & 2 & 100,0 & 100,0 & \\
\hline
\end{tabular}

Fuente. Datos procesados mediante el software SPSS 24 y Excel

Elaborado: Por el responsable de la investigación. 


\section{Figura $\mathbf{N}^{\circ} 3$}

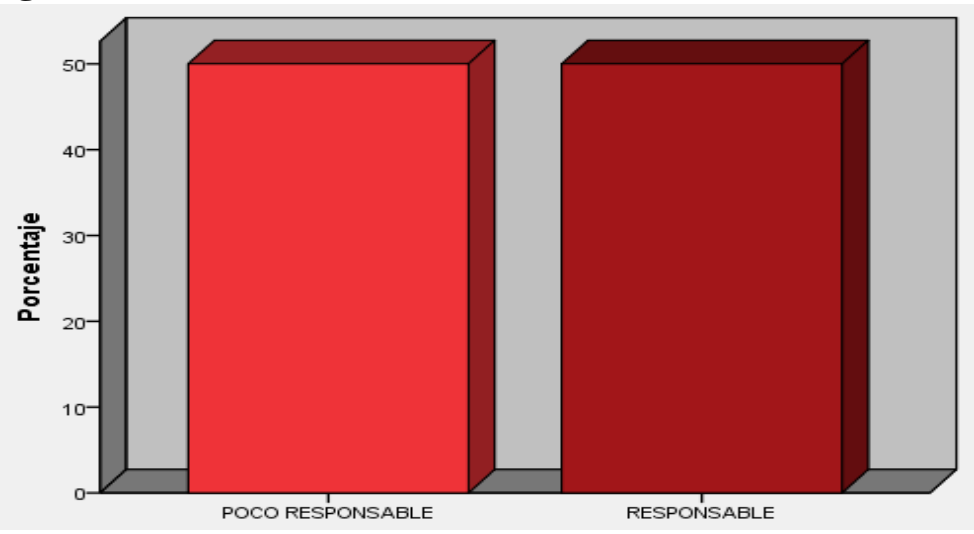

Variable: Evaluación del Desempeño Docente DIMENSIÓN: Estrategia Didáctica - Resultados Encuesta Docentes

\section{Cuadro $N^{\circ} 4$}

\begin{tabular}{|c|c|c|c|c|c|}
\hline \multicolumn{2}{|c|}{} & Frecuencia & Porcentaje & Porcentaje válido & $\begin{array}{c}\text { Porcentaje } \\
\text { acumulado }\end{array}$ \\
\hline \multirow{4}{*}{ Válido } & MALA & 2 & 6,7 & 6,7 & 6,7 \\
\cline { 2 - 6 } & REGULAR & 12 & 40,0 & 40,0 & 46,7 \\
\cline { 2 - 6 } & BUENA & 16 & 53,3 & 53,3 & 100,0 \\
\cline { 2 - 6 } & Total & 30 & 100,0 & 100,0 & \\
\hline
\end{tabular}

Fuente. Datos procesados mediante el software SPSS 24 y Excel

Elaborado: Por el responsable de la investigación.

\section{Figura $\mathbf{N}^{\circ} 4$}

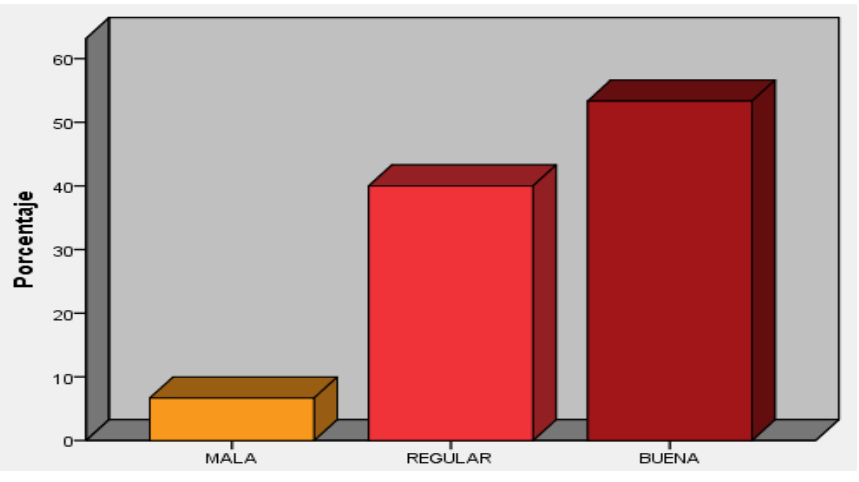

\section{DIMENSIÓN: Capacidades Pedagógicas - Resultados Encuesta Docentes}

\section{Cuadro $\mathbf{N}^{\circ} 5$}

\begin{tabular}{|c|l|c|c|c|c|}
\hline \multicolumn{2}{|c|}{} & Frecuencia & Porcentaje & Porcentaje válido & $\begin{array}{c}\text { Porcentaje } \\
\text { acumulado }\end{array}$ \\
\hline \multirow{4}{*}{ Válido } & MALAS & 2 & 6,7 & 6,7 & 6,7 \\
\cline { 2 - 6 } & REGULARES & 8 & 26,7 & 26,7 & 33,3 \\
\cline { 2 - 6 } & BUENAS & 20 & 66,7 & 66,7 & 100,0 \\
\cline { 2 - 6 } & Total & 30 & 100,0 & 100,0 & \\
\hline
\end{tabular}

Fuente. Datos procesados mediante el software SPSS 24 y Excel

Elaborado: Por el responsable de la investigación. 


\section{Figura $\mathbf{N}^{\circ} 5$}

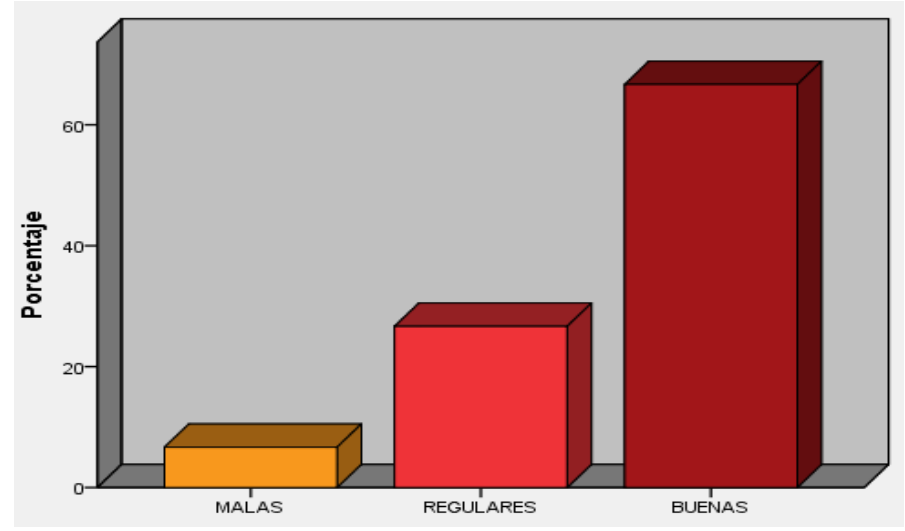

\section{Cuadro $\mathbf{N}^{\circ} 6$}

\begin{tabular}{|c|l|c|c|c|c|}
\hline \multicolumn{2}{|c|}{} & Frecuencia & Porcentaje & $\begin{array}{c}\text { Porcentaje } \\
\text { válido }\end{array}$ & $\begin{array}{c}\text { Porcentaje } \\
\text { acumulado }\end{array}$ \\
\hline \multirow{5}{*}{ Válido } & $\begin{array}{l}\text { NADA } \\
\text { RESPONSABLE }\end{array}$ & 4 & 13,3 & 13,3 & 13,3 \\
\cline { 2 - 6 } & $\begin{array}{l}\text { POCO } \\
\text { RESPONSABLE }\end{array}$ & 8 & 26,7 & 26,7 & 40,0 \\
\cline { 2 - 6 } & RESPONSABLE & 18 & 60,0 & 60,0 & 100,0 \\
\cline { 2 - 6 } & Total & 30 & 100,0 & 100,0 & \\
\hline
\end{tabular}

Fuente. Datos procesados mediante el software SPSS 24 y Excel

Elaborado: Por el responsable de la investigación.

\section{Figura $\mathbf{N}^{\circ} 6$}

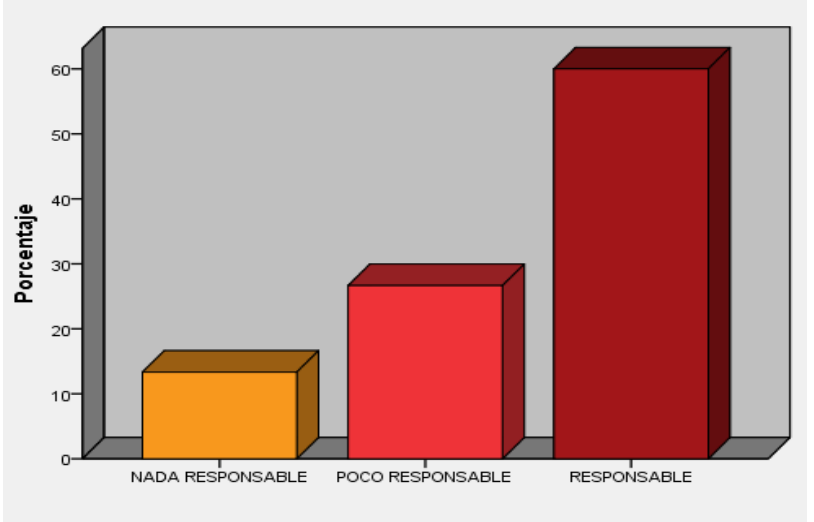

Variable: Evaluación del Desempeño Docente DIMENSIÓN: Estrategia Didáctica - Resultados Encuesta Estudiantes Cuadro $\mathbf{N}^{\circ} 7$

\begin{tabular}{|l|l|l|l|l|l|}
\hline \multicolumn{2}{|l|}{} & Frecuencia & Porcentaje & $\begin{array}{l}\text { Porcentaje } \\
\text { válido }\end{array}$ & $\begin{array}{l}\text { Porcentaje } \\
\text { acumulado }\end{array}$ \\
\hline \multirow{3}{*}{ Válido } & MALA & 15 & 18,8 & 18,8 & 18,8 \\
\cline { 2 - 6 } & REGULAR & 16 & 20,0 & 20,0 & 38,8 \\
\cline { 2 - 6 } & BUENA & 49 & 61,3 & 61,3 & 100,0 \\
\cline { 2 - 6 } & Total & 80 & 100,0 & 100,0 & \\
\hline
\end{tabular}

Fuente. Datos procesados mediante el software SPSS 24 y Excel.

Elaborado: Por el responsable de la investigación. 


\section{Figura $\mathbf{N}^{\circ} 7$}

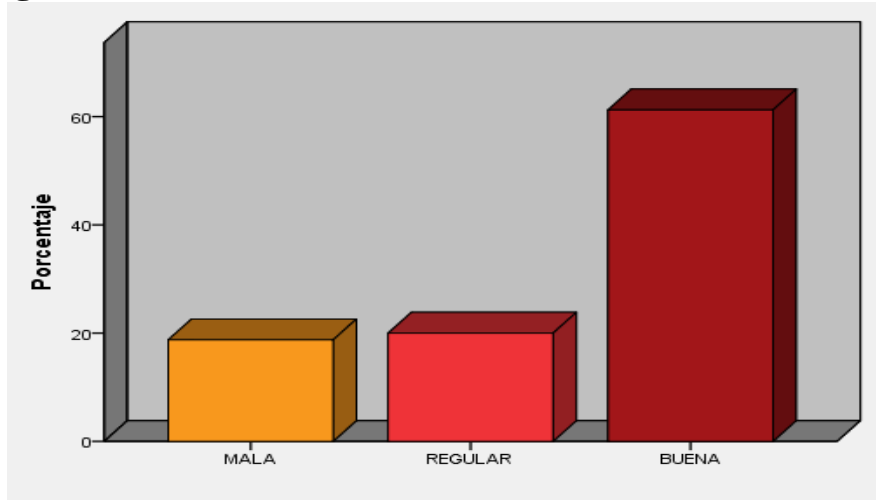

\section{DIMENSIÓN: Capacidades Pedagógicas - Resultados Encuesta Estudiantes} Cuadro $\mathbf{N}^{\circ} 8$

\begin{tabular}{|l|l|l|l|l|l|}
\hline \multicolumn{2}{|c|}{} & Frecuencia & Porcentaje & $\begin{array}{l}\text { Porcentaje } \\
\text { válido }\end{array}$ & $\begin{array}{l}\text { Porcentaje } \\
\text { acumulado }\end{array}$ \\
\hline \multirow{4}{*}{ Válido } & MALAS & 6 & 7,5 & 7,5 & 7,5 \\
\cline { 2 - 6 } & REGULARES & 33 & 41,3 & 41,3 & 48,8 \\
\cline { 2 - 6 } & BUENAS & 41 & 51,3 & 51,3 & 100,0 \\
\cline { 2 - 6 } & Total & 80 & 100,0 & 100,0 & \\
\hline
\end{tabular}

Fuente. Datos procesados mediante el software SPSS 24 y Excel Elaborado: Por el responsable de la investigación.

\section{Figura $\mathbf{N}^{\circ} 8$}

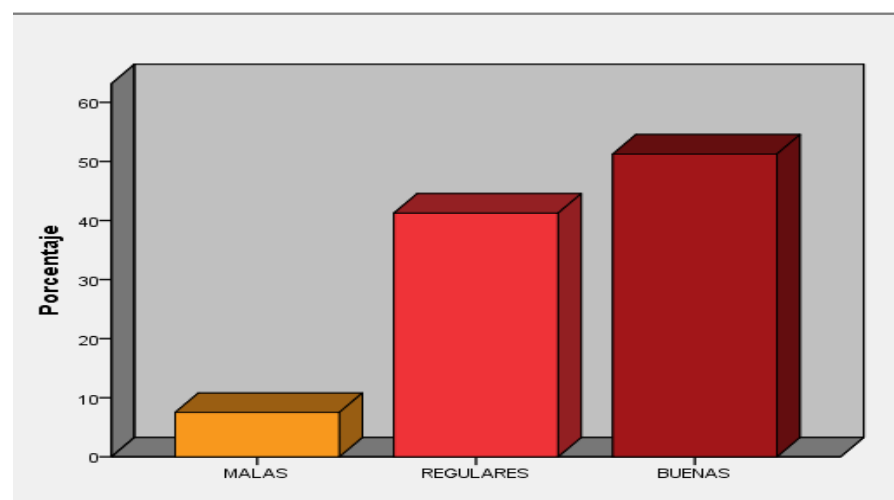

DIMENSIÓN: Responsabilidad de Funciones Laborales - Resultados Encuesta Estudiantes

\section{Cuadro $\mathbf{N}^{\circ} 9$}

\begin{tabular}{|l|l|l|l|l|l|}
\hline \multicolumn{2}{|c|}{} & Frecuencia & Porcentaje & $\begin{array}{l}\text { Porcentaje } \\
\text { válido }\end{array}$ & $\begin{array}{l}\text { Porcentaje } \\
\text { acumulado }\end{array}$ \\
\hline \multirow{5}{*}{ Válido } & $\begin{array}{l}\text { NADA } \\
\text { RESPONSABLE }\end{array}$ & 3 & 3,8 & 3,8 & 3,8 \\
\cline { 2 - 6 } & $\begin{array}{l}\text { POCO } \\
\text { RESPONSABLE }\end{array}$ & 27 & 33,8 & 33,8 & 37,5 \\
\cline { 2 - 7 } & RESPONSABLE & 50 & 62,5 & 62,5 & 100,0 \\
\cline { 2 - 7 } & Total & 80 & 100,0 & 100,0 & \\
\hline
\end{tabular}

Fuente. Datos procesados mediante el software SPSS 24 y Excel

Elaborado: Por el responsable de la investigación. 


\section{Figura $\mathbf{N}^{\circ} \mathbf{9}$}

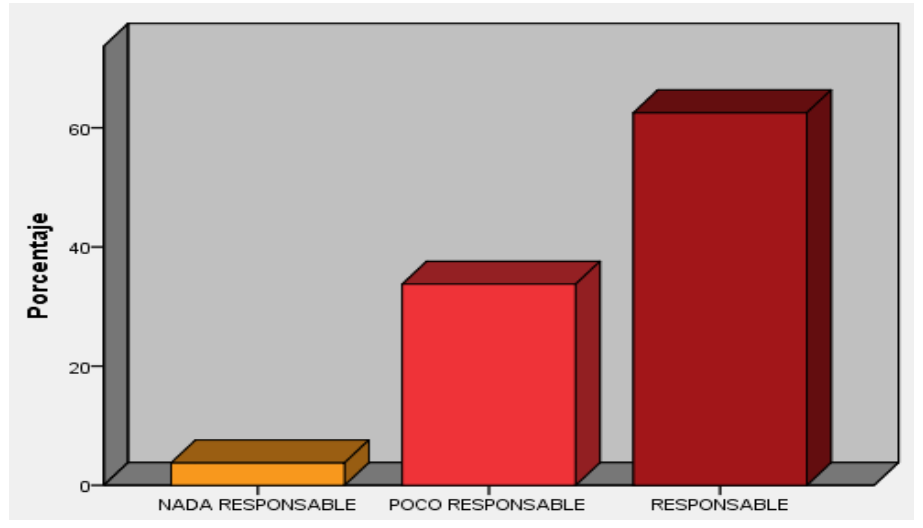

Variable: Habilidades para la vida

DIMENSIÓN: Habilidades Sociales - Resultados Encuesta Estudiantes

\section{Cuadro $\mathbf{N}^{\circ} 10$}

\begin{tabular}{|l|l|l|l|l|l|}
\hline \multicolumn{2}{|c|}{} & Frecuencia & Porcentaje & $\begin{array}{l}\text { Porcentaje } \\
\text { válido }\end{array}$ & $\begin{array}{l}\text { Porcentaje } \\
\text { acumulado }\end{array}$ \\
\hline \multirow{4}{*}{ Válido } & MALAS & 8 & 10,0 & 10,0 & 10,0 \\
\cline { 2 - 6 } & REGULARES & 27 & 33,8 & 33,8 & 43,8 \\
\cline { 2 - 6 } & BUENAS & 45 & 56,3 & 56,3 & 100,0 \\
\cline { 2 - 6 } & Total & 80 & 100,0 & 100,0 & \\
\hline
\end{tabular}

Fuente. Datos procesados mediante el software SPSS 24 y Excel Elaborado: Por el responsable de la investigación.

\section{Figura $\mathrm{N}^{\circ} 10$}

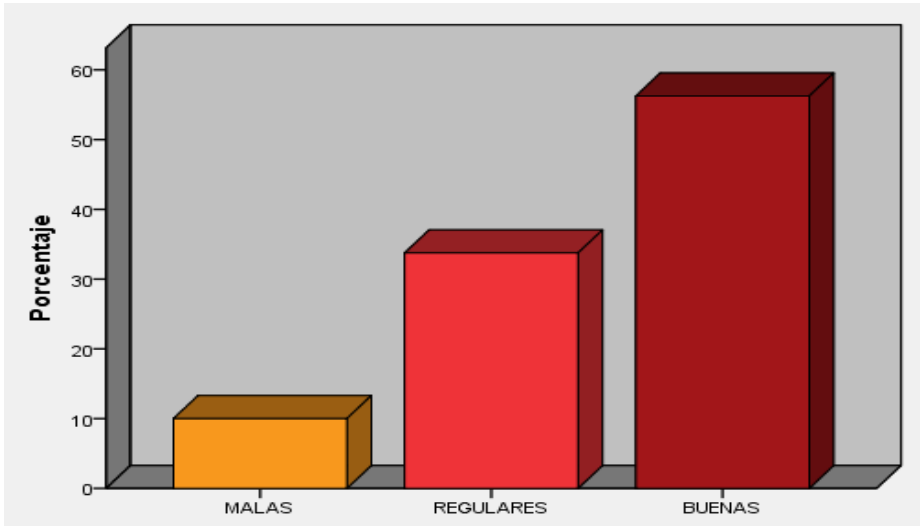

\section{Dimensión: Habilidades Cognitivas - Resultados Encuesta Estudiantes} Cuadro $\mathbf{N}^{\circ} 11$

\begin{tabular}{|l|l|l|l|l|l|}
\hline \multicolumn{2}{|c|}{} & Frecuencia & Porcentaje & $\begin{array}{l}\text { Porcentaje } \\
\text { válido }\end{array}$ & $\begin{array}{l}\text { Porcentaje } \\
\text { acumulado }\end{array}$ \\
\hline \multirow{4}{*}{ Válido } & MALAS & 24 & 30,0 & 30,0 & 30,0 \\
\cline { 2 - 6 } & REGULARES & 47 & 58,8 & 58,8 & 88,8 \\
\cline { 2 - 6 } & BUENAS & 9 & 11,3 & 11,3 & 100,0 \\
\cline { 2 - 6 } & Total & 80 & 100,0 & 100,0 & \\
\hline
\end{tabular}

Fuente. Datos procesados mediante el software SPSS 22 y Excel Elaborado: Por el responsable de la investigación. 


\section{Figura $\mathbf{N}^{\circ} 11$}

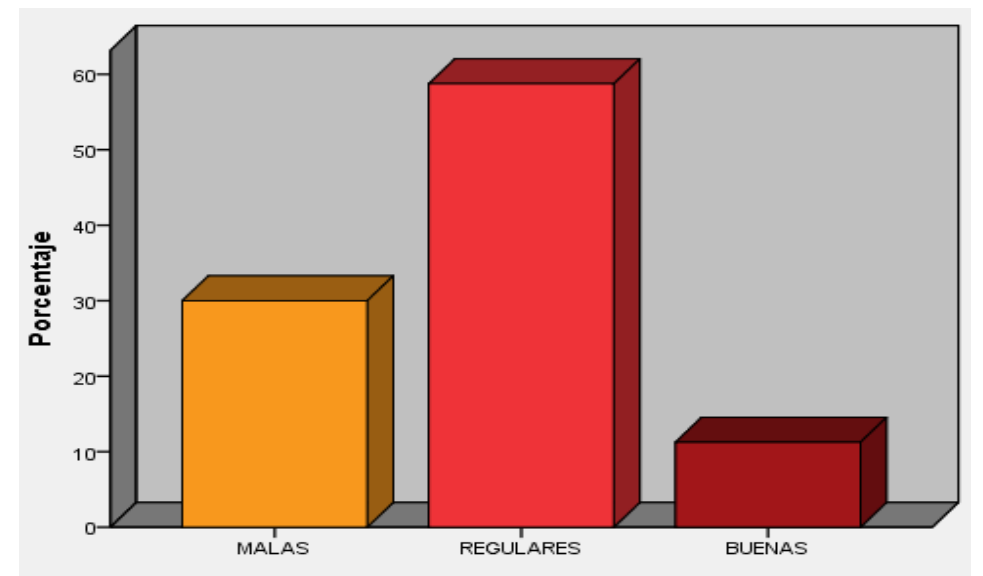

Dimensión: Habilidades para manejar conflictos - Resultados Encuesta Estudiantes

\section{Cuadro $\mathbf{N}^{\circ} 12$}

\begin{tabular}{|l|l|l|l|l|l|}
\hline \multicolumn{2}{|c|}{} & Frecuencia & Porcentaje & $\begin{array}{l}\text { Porcentaje } \\
\text { válido }\end{array}$ & $\begin{array}{l}\text { Porcentaje } \\
\text { acumulado }\end{array}$ \\
\hline \multirow{4}{*}{ Válido } & MALAS & 7 & 8,8 & 8,8 & 8,8 \\
\cline { 2 - 6 } & REGULARES & 46 & 57,5 & 57,5 & 66,3 \\
\cline { 2 - 6 } & BUENAS & 27 & 33,8 & 33,8 & 100,0 \\
\cline { 2 - 6 } & Total & 80 & 100,0 & 100,0 & \\
\hline
\end{tabular}

Fuente: Datos procesados mediante el software SPSS 24 y Excel

Elaborado: Por el responsable de la investigación.

\section{Figura $\mathbf{N}^{\circ} 12$}

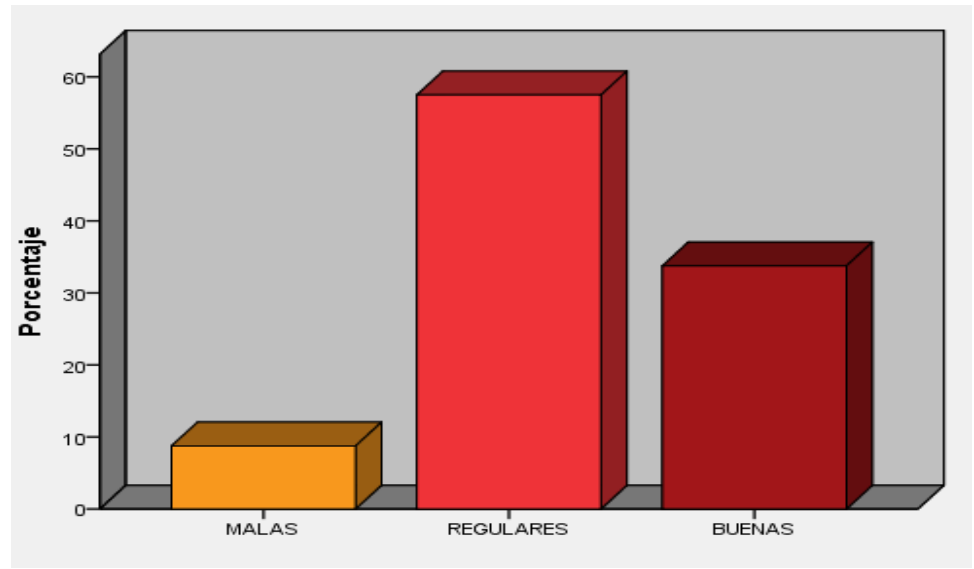

\section{Planteamiento de la Hipótesis General}

H0: La evaluación del desempeño docente no influye significativamente en las habilidades para la vida de los estudiantes del nivel secundario de la Institución Educativa Privada PAMER, Los Olivos - Lima Norte, 2017.

Ha: La evaluación del desempeño docente influye significativamente en las habilidades para la vida de los estudiantes del nivel secundario de la Institución Educativa Privada PAMER, Los Olivos - Lima Norte, 2017. 


\section{Journal of the Academy $|145|$}

\section{Regla de decisión}

La contrastación de hipótesis utilizó la correlación de Rho Spearman entre la variable evaluación del desempeño docente y la variable habilidades para la vida.

Figura $\mathbf{N}^{\circ} 13$

Matriz de correlación entre las variables evaluación del desempeño docente y habilidades para la vida.

\begin{tabular}{|c|c|c|c|c|}
\hline & & & $\begin{array}{c}\text { Evaluación del } \\
\text { desempeño docente }\end{array}$ & $\begin{array}{l}\text { Habilidades para } \\
\text { la vida }\end{array}$ \\
\hline \multirow{6}{*}{$\begin{array}{l}\text { Rho de } \\
\text { Spearman }\end{array}$} & \multirow{3}{*}{$\begin{array}{l}\text { Evaluación del } \\
\text { desempeño } \\
\text { docente }\end{array}$} & $\begin{array}{l}\text { Coeficiente de } \\
\text { correlación }\end{array}$ & 1,000 & $0,783^{* *}$ \\
\hline & & Sig. (bilateral) & & 0,009 \\
\hline & & $\mathrm{N}$ & 112 & 112 \\
\hline & \multirow{3}{*}{$\begin{array}{l}\text { Habilidades para } \\
\text { la } \\
\text { vida }\end{array}$} & $\begin{array}{l}\text { Coeficiente de } \\
\text { correlación }\end{array}$ & $0,783^{* *}$ & 1,000 \\
\hline & & Sig. (bilateral) & 0,009 & \\
\hline & & $\mathrm{N}$ & 112 & 112 \\
\hline
\end{tabular}

**. La correlación es significativa en el nivel 0,01 (2 colas).

El análisis estadístico efectuado con el Rho de Spearman por tratarse de variables arroja una relación de $\mathrm{r}=0,783$ (donde $\mathrm{p}<$ de 0,05 ) entre evaluación del desempeño docente y las habilidades para la vida en los estudiantes. Al obtenerse una significancia bilateral de 0,009 la misma que se encuentra dentro del valor permitido $(0,05)$ se rechaza la hipótesis nula y se concluye que la evaluación del desempeño docente influye significativamente en las habilidades para la vida en los estudiantes del nivel secundario de la Institución Educativa Privada PAMER, Los Olivos - Lima Norte, 2017. Estos resultados se dan en un $99 \%$ de intervalo de confianza.

\section{Planteamiento de hipótesis específicas}

\section{Hipótesis específica 01}

H0: No Existe influencia significativa entre las estrategias didácticas con las habilidades para la vida de los estudiantes del nivel secundario de la Institución Educativa Privada PAMER, Los Olivos - Lima Norte, 2017.

H1: Existe influencia significativa entre las estrategias didácticas con las habilidades para la vida de los estudiantes del nivel secundario de la Institución Educativa Privada PAMER, Los Olivos - Lima Norte, 2017.

\section{Figura $\mathbf{N}^{\circ} 14$}

Matriz de correlación de la dimensión estrategias didácticas y la variable habilidades para la vida.

\begin{tabular}{|c|c|c|c|c|}
\hline & & & $\begin{array}{l}\text { Estrategias } \\
\text { didácticas }\end{array}$ & $\begin{array}{c}\text { Habilidades para la } \\
\text { vida }\end{array}$ \\
\hline & Fstrategiac didácticos & $\begin{array}{l}\text { Coeficiente de } \\
\text { correlación }\end{array}$ & 1,000 &, $736^{* *}$ \\
\hline & Estrategias didacticas & Sig. (bilateral) & & ,008 \\
\hline & & $\mathrm{N}$ & 112 & 112 \\
\hline Rho de Spearman & Habilidades para la & $\begin{array}{l}\text { Coeficiente de } \\
\text { correlación }\end{array}$ &, $36^{* *}$ & 1,000 \\
\hline & vida & Sig. (bilateral) & ,008 & \\
\hline & & $\mathrm{N}$ & 112 & 112 \\
\hline
\end{tabular}

**. La correlación es significativa en el nivel 0,01 (2 colas).

Los resultados del análisis estadístico proyectan una relación de $\mathrm{r}=0,736$ (donde $\mathrm{p}<\mathrm{de} 0,05$ ) entre las estrategias didácticas y con las habilidades para la vida en los estudiantes del nivel secundario. Al obtenerse una significancia bilateral de 0,008 la misma que se encuentra dentro del valor permitido 


\section{Journal of the Academy $|146|$}

$(0,05)$ se rechaza la segunda hipótesis específica y se concluye que existe influencia significativa entre las estrategias didácticas con las habilidades para la vida en los estudiantes del nivel secundario de la Institución Educativa Privada PAMER, Los Olivos - Lima Norte, 2017. Estos resultados se dan en un $99 \%$ de intervalo de confianza.

Hipótesis específica 02

H0: No Existe influencia significativa entre las capacidades pedagógicas con las habilidades para la vida en los estudiantes del nivel secundario de la Institución Educativa Privada PAMER, Los Olivos Lima Norte, 2017.

H2: Existe influencia significativa entre las capacidades pedagógicas con las habilidades para la vida en los estudiantes del nivel secundario de la Institución Educativa Privada PAMER, Los Olivos - Lima Norte, 2017.

\section{Figura $\mathbf{N}^{\circ} 15$}

Matriz de correlación de la dimensión capacidades pedagógicas y la variable habilidades para la vida.

\begin{tabular}{|c|c|c|c|c|}
\hline & & & $\begin{array}{l}\text { Capacidades } \\
\text { pedagógicas }\end{array}$ & $\begin{array}{l}\text { Habilidades } \\
\text { para la vida }\end{array}$ \\
\hline \multirow{6}{*}{$\begin{array}{l}\text { Rho de } \\
\text { Spearman }\end{array}$} & \multirow{3}{*}{$\begin{array}{l}\text { Capacidades } \\
\text { pedagógicas }\end{array}$} & $\begin{array}{l}\text { Coeficiente de } \\
\text { correlación }\end{array}$ & 1,000 &, $716^{* *}$ \\
\hline & & Sig. (bilateral) & & ,008 \\
\hline & & $\mathrm{N}$ & 112 & 112 \\
\hline & \multirow{3}{*}{$\begin{array}{l}\text { Habilidades para la } \\
\text { vida }\end{array}$} & $\begin{array}{l}\text { Coeficiente de } \\
\text { correlación }\end{array}$ &, $716^{* *}$ & 1,000 \\
\hline & & Sig. (bilateral) & ,008 & \\
\hline & & $\mathrm{N}$ & 112 & 112 \\
\hline
\end{tabular}

**. La correlación es significativa en el nivel 0,01 ( 2 colas).

Los resultados del análisis estadístico arrojan una relación de $\mathrm{r}=0,716$ (donde $\mathrm{p}<\mathrm{de} 0,05$ ) entre las capacidades pedagógicas con las habilidades para la vida en los estudiantes. Al obtenerse una significancia bilateral de 0,008 la misma que se encuentra dentro del valor permitido $(0,05)$ se rechaza la segunda hipótesis específica y se concluye que existe influencia significativa entre las capacidades pedagógicas con las habilidades para la vida en los estudiantes del nivel secundario de la Institución Educativa privada PAMER, Los Olivos - Lima Norte, 2017. Estos resultados se dan en un $99 \%$ de intervalo de confianza.

\section{Hipótesis específica 03}

H0: No Existe influencia significativa entre la responsabilidad de funciones laborales con las habilidades para la vida en los estudiantes del nivel secundario de la Institución Educativa Privada PAMER, Los Olivos - Lima Norte, 2017.

H3: Existe influencia significativa entre la responsabilidad de funciones laborales con las habilidades para la vida en los estudiantes del nivel secundario de la Institución Educativa Privada PAMER, Los Olivos - Lima Norte, 2017.

\section{Figura $\mathbf{N}^{\circ} 16$}

Matriz de correlación de la dimensión responsabilidad de funciones laborales y la variable habilidades para la vida.

\begin{tabular}{|l|l|c|c|c|}
\hline \multicolumn{2}{|l|}{} & $\begin{array}{l}\text { Responsabilidad de } \\
\text { funciones laborales }\end{array}$ & $\begin{array}{c}\text { Habilidades } \\
\text { para la vida }\end{array}$ \\
\hline \multirow{2}{*}{$\begin{array}{l}\text { Rho de } \\
\text { Spearman }\end{array}$} & $\begin{array}{l}\text { Responsabilidad de } \\
\text { funciones laborales }\end{array}$ & $\begin{array}{l}\text { Coeficiente de } \\
\text { correlación }\end{array}$ & 1,000 &, $722^{* *}$ \\
\cline { 3 - 5 } & Sig. (bilateral) &. &, 008 \\
\cline { 3 - 5 } & N & 112 & 112 \\
\hline
\end{tabular}


Journal of the Academy | $147 \mid$

\begin{tabular}{|l|l|l|c|c|}
\hline \multirow{2}{*}{ Habilidades para la } & $\begin{array}{l}\text { Coeficiente de } \\
\text { vida }\end{array}$ &, $722^{* *}$ & 1,000 \\
\cline { 2 - 4 } & $\begin{array}{l}\text { Sig. (bilateral) } \\
\text { Nigén }\end{array}$ &, 008 &. \\
\cline { 2 - 4 } & $\mathrm{N}$ & 112 & 112 \\
\hline
\end{tabular}

**. La correlación es significativa en el nivel 0,01.

Los resultados del análisis estadístico arrojan una relación de $\mathrm{r}=0,722$ (donde $\mathrm{p}<$ de 0,05 ) entre la responsabilidad de funciones laborales con las habilidades para la vida en los estudiantes del nivel secundario. Al obtenerse una significancia bilateral de 0,008 la misma que se encuentra dentro del valor permitido $(0,05)$ se rechaza la tercera hipótesis específica y se concluye que eexiste influencia significativa entre la responsabilidad de funciones laborales con las habilidades para la vida de los estudiantes del nivel secundario de la Institución Educativa privada PAMER, Los Olivos- Lima Norte, 2017. Estos resultados se dan en un $99 \%$ de intervalo de confianza.

\section{CONCLUSIONES}

El estudio realizado ha determinado la influencia que existe entre la evaluación del desempeño docente y las habilidades para la vida de los estudiantes del nivel secundario, a un nivel de significancia del 5\% y un valor $\mathrm{p}=0.000<0.05$, se rechaza la Hipótesis Nula y se acepta la Hipótesis Alterna que sustenta que es significativa y alcanza un nivel de 0.783 que corresponde a un nivel correlación positiva moderada. Por lo tanto, esto quiere decir que los docentes manejan estrategias didácticas, capacidades pedagógicas y tienen responsabilidad de sus funciones laborales.

Se ha analizado que las estrategias didácticas influyen en las habilidades para la vida de los estudiantes del nivel secundario, con el nivel de 0.736 que corresponde a un nivel correlación positiva moderada. Por lo tanto, se puede afirmar que los docentes hacen buen uso de la metodología en los usos de materiales didácticos en el desarrollo de sus actividades para mejorar las habilidades cognitivas del estudiante.

Se puede afirmar que las capacidades pedagógicas influyen en las habilidades para la vida de los estudiantes del nivel secundario, es decir, con una correlación significativa de 0.716 que corresponde a un nivel correlación positiva. Entonces se puede afirmar que los docentes elaboran sesiones de aprendizaje, demuestran conocimientos actualizados en el área que enseñan, crean un clima propicio para el aprendizaje y se capacitan constantemente.

Asimismo, en la tercera dimensión se observó que la responsabilidad de funciones laborales influye en las habilidades para la vida de los estudiantes con una significancia del nivel de 0.722 que corresponde a un nivel correlación positiva. Por ello, se puede afirmar que los docentes mantienen la responsabilidad de sus funciones laborales con las obligaciones y la programación curricular y mantiene sus actitudes en la evaluación con los estudiantes.

\section{REFERENCIAS BIBLIOGRÁFICAS}

Ávalos, H. (2015). Programa Habilidades Para La Vida En La Comuna De Pudahuel: Revisión Desde La Práctica Profesional. Universidad de Chile, Facultad de Ciencias Sociales.

Bravo, A., Martínez Ruíz, V. y Mantilla, L. (2003). Habilidades para la vida: Una propuesta educativa para convivir mejor. Bogotá: FYA de Colombia.

Cerda, H. (2003). La nueva evaluación educativa. Bogotá: Cooperativa Editorial Magisterio. 


\section{Journal of the Academy $|148|$}

Choque, R. (2007). Eficacia del programa educativo de habilidades para la vida en adolescentes de una institución educativa del distrito de Huancavelica, 2006 (Tesis de Maestría). Universidad Peruana Cayetano Heredia: Escuela de Postgrado Víctor Alzamora Castro, Lima.

García, J. (2007). Relación entre la Ejecución Curricular y el Desempeño Docente, según los estudiantes de la Facultad de educación de la Universidad Nacional Federico Villarreal (Tesis de Maestría). Universidad Nacional Mayor de San Marcos, Lima.

Hernández, S., Fernández, A. y Baptista, A. (2014). Metodología de la Investigación. México: Editorial Mc Graw Hill.

Mangrulkar, L., Vince, C. y Posner, M. (2001). Enfoque de habilidades para la vida para un desarrollo saludable de niños y adolescentes. Washington: Organización Panamericana de la Salud.

Marcano, L. (2006). Modelo de evaluación del desempeño profesional docente República de Cuba. Comisión Nacional de Grados Científicos (Tesis de Doctorado). Universidad Pedagógica Félix Varela, La Habana.

Mateo, J. (1998). La evaluación educativa. En Enciclopedia General de la Educación. Barcelona: Océano.

Mateo, J. (2000). La evaluación educativa, su práctica y otras metáforas. Barcelona: Ed. Horsori.

Mejía, E. (2005). Metodología de la Investigación Científica. Lima: Centro de Producción Editorial e Imprenta de la UNMSM.

Mejia, E. (2005). Técnicas e instrumentos de investigación científica. Lima: Centro de Producción Editorial e Imprenta de la UNMSM.

Ministerio de Educación. (2007). Sistema de Evaluación de Desempeño Docente Programa de educación en áreas rurales PEAR. Lima: MED, Gestión pedagógica.

Montenegro, I. (2003). Evaluación del desempeño docente. Santa Fe de Bogotá: Cooperativa Editorial Magisterio.

OMS (1993). Habilidades para la Vida en las Escuelas. Ginebra: División de salud mental - OMS.

Preciado, S. (2014). Fortalecimiento de habilidades para la vida como factores psicosociales en la educación para la resiliencia. (Tesis de Licenciatura). Universidad Nacional abierta y a distancia UNAD. Escuela de ciencias sociales, artes y humanidades programa de psicología, Sogamoso.

Tafur, R. (1995). La Tesis Universitaria. La tesis doctoral, la tesis de maestría, el informe y la monografía. Lima: Editorial Mantaro.

Valdés, H. (2000). Evaluación del Desempeño Docente. Ponencia presentada en el Encuentro Iberoamericano sobre Evaluación del Desempeño Docente, Ciudad de México, México.

Villa, Aurelio y otros autores (1995). Perspectivas y problemas de la función docente. Editorial Narcea, Madrid, España. 